\section{Primary health care psychiatry}

Primary Health Care Psychiatry: A Practical Guide for Southern Africa. Ed. by Sean Exner Baumann. Cape Town: Juta. Pp. 856. ISBN 978 D 702172984.

Much needs to be done to improve psychiatric care in South Africa. Resources need to be developed, particularly in rural areas, and psychiatric services need to be better integrated into primary health care services. This process will include the intensive training of mental health care workers. If we look at the training of primary care physicians, their rotation during the 2-year internship falls short in psychiatry. It is not seen as a mainstream rotation point like disciplines such as surgery or internal medicine. This fact, as well as a more student-centred approach and emphasis on self-learning in medical curricula, make the search for an appropriate prescribed textbook in psychiatry an important issue.

It was with the above in mind that I reviewed this book. It provides a broad conceptual framework for understanding the scope and practice of mental health, and is a new book rather than simply a second edition of an earlier volume, Psychiatry and Primary Health Care. The great majority of chapters have been rewritten by new authors from across South Africa, who have recognised expertise and clinical experience in their fields. Nine new chapters have been added and further additions have been made to the appendices. Diagnostic criteria have been included in the clinical chapters to make the text more useful to readers. The broad aim is to update and expand the contents of the first book.

A problem-based approach has been maintained, and extensive use is made of personal accounts and care vignettes. These both illustrate clinical points and emphasise the gulf between objective, clinical accounts and the subjective intense experience of those who suffer from mental illness. Much of value can be learned from such first-person perspectives as the 'mother's story' in the section on schizophrenia.

The book is divided into the following sections:

Section 1: An introduction to primary care in southern Africa. Language and cultural issues are addressed and local categories or idioms of distress are described.

Section 2: A scheme of history-taking and the mental state examinations, diagnosis and classification at the primary level is described. A problem-based approach is followed. It is not assumed that a diagnosis is required in order to treat.
Section 3: Problems likely to be encountered in primary care with treatment plans. The synopsis at the end of each chapter provides the clinician with a practical scheme for assessment and management that may be read at a glance. Guidelines for referral management problems pertinent to the local context include topics such as coping with trauma, coping with loss, alcohol and other substance-abuse disorders, HIV and mental health, and the phenomenon of burnout.

Section 4: This includes the more common major clinical syndromes.

Section 5: This deals with psychiatry and the law and philosophical and ethical issues that pertain to psychiatry. Important for the primary care clinician is information about the Mental Health Care Act and the procedures to be followed for the admission and treatment of psychiatric patients

Section 6: Pharmacological and non-pharmacological methods of treatment appropriate at the primary level of care are described.

The appendices include a glossary of psychiatric terms, guidelines for the pharmacological treatment of common problems in primary care psychiatry, and basic schemes for the management of violent patients, relapses of psychotic illnesses, alcohol and substanceabuse related problems and suicidal behaviour.

References and recommendations for further reading are to be found at the end of the book.

There are important challenges facing psychiatry in southern Africa at the beginning of the 21 st century. These challenges create the opportunity to develop models of care and service delivery that are just, effective and appropriate to the southern African context.

This book has been long overdue. It fulfils a need for practical and relevant information, based on the best available evidence, and provided in clear and accessible language unfettered by professional jargon. Sean Baumann, as editor, and an outstanding group of contributors have provided an excellent psychiatric text reference for the southern African context.

\section{J L Roos}

Department of Psychiatry University of Pretoria 